\title{
Social Detachment Influenced Muscle Mass and Strength during the COVID-19 Pandemic in Japanese Community-Dwelling Older Women
}

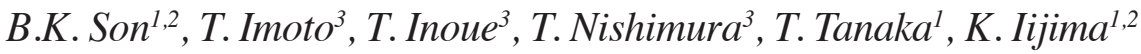

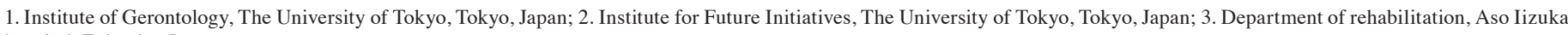 \\ hospital, Fukuoka, Japan

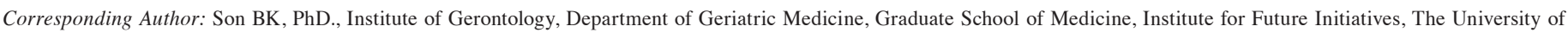 \\ Tokyo, Tokyo, Japan, 7-3-1 Hongo, Bunkyo-ku, Tokyo 113-8655, Japan. Phone: 8135800 6534, Fax: 813 5800 8837, E-mail: son@iog.u-tokyo.ac.jp
}

\begin{abstract}
Social detachment due to coronavirus disease (COVID-19) has caused a decline in physical activity, leading to sarcopenia and frailty in older adults. This study aimed to compare muscle mass, strength, and function values in older women before and after the first wave of the COVID-19 pandemic (April-May 2020). Furthermore, changes in muscle measures across women who experienced different levels of impact on their social participation due to the COVID-19 pandemic were examined. Muscle mass (total, trunk, and appendicular muscle), grip strength, oral motor skills, social interactions (social network and participation), and social support were assessed in 46 Japanese community-dwelling older women (mean, 77.5 y; range 66-93 y) before and after the first wave of the COVID-19 pandemic. Trunk muscle mass significantly decreased after the first wave of the pandemic. When comparing changed values between the enhanced/maintained and reduced group during the pandemic, significant group difference was observed in trunk muscular mass, grip strength, and oral motor skills. Intriguingly, those who enhanced social participation had a positive change of grip strength values, showing that social participation might influence muscle function during the COVID-19 pandemic.
\end{abstract}

Key words: COVID-19, trunk muscle mass, grip strength, social participation, older adults.

\section{Introduction}

evere acute respiratory syndrome coronavirus 2 (SARS-CoV-2), the causative virus of coronavirus disease 2019 (COVID-19), emerged in late 2019, and the disease and the complications arising due to this disease are a global public health challenge (1). To mitigate the spread of the infection, the Japanese government declared a nationwide state of emergency on April 16, 2020 and going outdoors was restricted by May 25, 2020. Despite the benefits of infection control by this restriction, many individuals, especially older adults, faced an increased risk for secondary health issues (2, 3 ). In particular, the onset/progression of sarcopenia (the loss of functional strength and skeletal muscle mass) and frailty (an age-related syndrome characterized by decreased reserve and resistance to stressors), two representative geriatric problems, was a major concern..

During the COVID-19 pandemic, social detachment has been reported to negatively affect not only mental health and social well-being, (4) but also physical activity (5) in older adults. In Japan, an association has recently been demonstrated between the decrease in frequency of socialization (defined as moving from one's residence to another place or region) and daily life, social life, and physical function in communitydwelling adults after the COVID-19 pandemic (6). Regarding physical activity, due to the social detachment caused by COVID-19 in 2020, the total time of physical activity (PA) in community-dwelling older adults has decreased to $40.0 \%$ over a period of one year, and $16.0 \%$ of the participants had a new incidence of frailty during this follow-up period (5).

Intriguingly, the decline in social function, including frequency of going outdoors, has been suggested to be directly related to the decline in oral functions (e.g. oral motor skills) and other physical functions (7). However, to examine the impact of COVID-19, quantitative measurement of skeletal and oral muscle did not perform. Additionally, there are limited studies examining the impact of COVID-19 on social participations and subsequently on muscular functions.

In this study, we compared muscle mass, strength, and function values in older women before and after the first wave of the COVID-19 pandemic. We also examined changes in muscle measures across women who experienced different levels of impact on their social participation due to the COVID19 pandemic.

\section{Methods}

\section{Participants}

Among the 58 community-dwelling older adults who participated in a frailty check program once every 6 months, we included 46 participants who underwent physical examination and completed a survey between both October 2019-February 2020 (before the first wave of COVID-19), and July-September 2020 (after the first wave of COVID-19) in Iitsuka city, Fukuoka, Japan. This frailty check program was developed based on the evidence of the Kashiwa cohort study starting 2012, which was conducted in Kashiwa city, Chiba, Japan and the participants were randomly selected and invited the elderly aged 65 years and over who were not eligible for long-term care, to check frailty once every 6 months continuously (8). 
We excluded participants with missing data, and all four male participants. This study was conducted in accordance with the guidelines of the Declaration of Helsinki, and the study protocol was reviewed and approved by the Ethics Committee of Life Science (\#19-320).

\section{Physical examination}

Total, trunk, and appendicular muscle mass values were measured via bioelectrical impedance analysis using a body composition analyzer (Inner scan dual RD800, TANITA, Tokyo, Japan). Muscle strength was assessed via handgrip strength, which was measured using a digital grip strength dynamometer (Takei Scientific Instruments, Niigata, Japan) (9). To evaluate oral motor skills and speech ability, we used oral diadochokinesis (10). In detail, the repetition of monosyllables $/ \mathrm{Ta} /$ and $/ \mathrm{Ka} /$ as quickly as possible for $5 \mathrm{~s}$ was measured with an oral function measuring device (Kenkou-Kun handy, Takei Scientific Instruments Co., Ltd., Niigata, Japan) (11).

\section{Questionnaire}

To assess the frequency of going out among the participants, the "yes/no" question "Has your frequency of going out declined, compared with that last year?" was included. Exercise habits (once a day for $>30$ min performed $>$ twice per week and since $>1 \mathrm{y}$ ) and walking/physical activity ( $>1 \mathrm{~h}$ per day) before the first wave of COVID-19 were assessed the "yes/no" question.

To better understand the impact of COVID-19 on socialization, we assessed social interactions and social support using proper questionnaires. Social interactions were assessed by social network and social participation. Social network was assessed by the Lubben social network scale, a self-report measure of social network with family and friends (12). We used a short version of this scale with six items that comprises three items for family and three for friendships $(0-5$ points for each item, total 30 points). Social participation was surveyed based on participation in seven groups (senior, health/sport, learning, hobby, community activity, volunteer groups, and working groups with income), totaling to 7 points based on "yes/no" responses to questions regarding participation.

Social support was assessed via four questionnaires; two questionnaires assessed whether one had someone to counsel or help perform housework or shopping, and the other two questionnaires assessed whether one received counseling to perform housework or shopping; thus, totaling to 4 points for the yes/no responses.

\section{Statistical analysis}

We examined the effect of the COVID-19 pandemic by assessing muscular measurements before and after its first wave using a paired t-test and Wilcoxon rank-sum test, respectively, after confirmation of normal distribution by the Shapiro-Wilk test. Changes in individual physical parameters were assessed using the student t-test, Mann-Whitney U-test, and Jonckheere test after confirming normal distribution by the Shapiro-Wilk test and homogeneity of variance by the Levene test.

Trunk muscle mass index $\left(\mathrm{kg} / \mathrm{m}^{2}\right)$, ASM $(\mathrm{kg})$, ASMI $(\mathrm{kg} /$ $\mathrm{m}^{2}$ ), articulatory oral motor skill "Ta", "Ka" (times/s), social network, and social support were seen non-normal distribution. Data were analyzed using SPSS IBM statistics version 26 (IBM Japan, Tokyo, Japan). Values of $\mathrm{P}<0.05$ were considered significant in all analyses.

\section{Results}

This study included 46 older women who underwent physical assessments and answered questionnaires before and after the first wave of COVID-19. Their median age was 77.5 years. Before the first wave of COVID-19, $60.9 \%$ of the participants in this study had exercise habits (once a day for $>30$ min performed $>$ twice per week and since $>1 \mathrm{y}$ ) and $76.1 \%$ of the participants did walking/physical activity ( $>1 \mathrm{~h}$ per day). Due to the first wave of COVID-19, 65.2\% of participants answered that they had a lesser frequency of going out after the first wave compared with that of the previous year. Given that the frequency of going outdoors has been suggested to be a good predictor for incident disability of physical function in older adults $(13,14)$, the decrease in physical activity in our participants is conceivable.

In Table 1, muscular measures, social interactions, and social support are shown before and after the first wave of COVID19. Notably, after the first wave, trunk muscle mass values significantly decreased, and a slight decrease in total muscle mass was seen. Appendicular skeletal muscle (ASM) mass and the ASM index (ASMI) significantly increased.

Next, we further compared the changes (before value subtracted from after value) of each muscular and social parameter by dividing the participants into two groups: the first was an enhanced/maintained group with enhanced or maintained changes after the first wave of COVID-19, and the second was a reduced group with reduced changes. When compared between the two groups, trunk muscle mass (-1.63 $\pm 1.20 \mathrm{~kg}$ ) was significantly lower in the reduced group than in the enhanced/maintained group $(0.44 \pm 0.32 \mathrm{~kg}$, Table 1$)$. On the other hand, changes in ASM and ASMI values of enhanced/ maintained and reduced groups did not differ. Regarding muscle strength, a slight decrease in grip strength was observed after the first wave of COVID-19, but changes in values were significant in the reduced versus enhanced/maintained group $(-1.94 \pm 1.16 \mathrm{~kg}$ vs. $1.26 \pm 0.78 \mathrm{~kg}$, Table 1$)$. We also compared oral function using an oral motor skill test ("Ta" and "Ka"), because a decrease in oral function has been associated with muscular decline (7). We found that both oral motor skills "Ta" and "Ka" slightly, but not significantly, decreased after the first wave. Furthermore, as shown in Table 1, oral motor skill "Ka" values in the reduced group were lower than those of the enhanced/maintained group after the first wave.

When we examined the impact of COVID-19 on social network, social participation, and social support, compared with that before the first wave of COVID-19, social support significantly decreased after the first wave (Table 1). 
Table 1. Changes in muscle mass and strength, physical, oral and social functions in older adults before and after the COVID-19 pandemic

\begin{tabular}{|c|c|c|c|c|c|c|c|}
\hline & & \multicolumn{2}{|c|}{$\begin{array}{l}\text { COVID-19 pandemic } \\
\text { (1st wave)* }\end{array}$} & \multirow[t]{2}{*}{$p \neq$} & \multicolumn{2}{|c|}{ Changes after the 1 st wave $\dagger$} & \multirow[t]{2}{*}{ p\$ } \\
\hline & & Before & After & & Enhanced/ Maintained & Reduced & \\
\hline \multirow[t]{4}{*}{ Muscle mass } & Total muscle mass $(\mathrm{kg})$ & $32.6(3.2)$ & $31.9(4.0)$ & 0.257 & $0.71 \pm 0.53$ & $-0.87 \pm 0.71$ & 0.381 \\
\hline & Trunk muscle mass (kg) & $18.3(2.4)$ & $17.0(3.3)$ & $\mathrm{p}<0.001$ & $0.44 \pm 0.32$ & $-1.63 \pm 1.20$ & $\mathrm{p}<0.001$ \\
\hline & Trunk muscle mass index $\left(\mathrm{kg} / \mathrm{m}^{2}\right)$ & $8.1(0.4)$ & $7.6(0.7)$ & $\mathrm{p}<0.001$ & $0.18 \pm 0.12$ & $-0.69 \pm 0.50$ & $\mathrm{p}<0.001$ \\
\hline & $\mathrm{ASM}(\mathrm{kg})$ & $14.2(2.5)$ & $15.5(2.2)$ & $\mathrm{p}<0.001$ & $0.73 \pm 0.50$ & $-1.87 \pm 0.71$ & 0.486 \\
\hline Muscle strength & Grip strength (kg) & $21.7(4.8)$ & $21.4(4.8)$ & 0.150 & $1.26 \pm 0.78$ & $-1.94 \pm 1.16$ & 0.026 \\
\hline \multirow[t]{2}{*}{ Oral function } & Articulatory oral motor skill "Ta” (times/s) & $6.1(1.2)$ & $6.2(1.3)$ & 0.131 & $0.27 \pm 0.64$ & $-0.82 \pm 1.05$ & 0.116 \\
\hline & Articulatory oral motor skill "Ka”(times/s) & $5.8(0.9)$ & $5.8(1.1)$ & 0.143 & $0.43 \pm 0.39$ & $-0.99 \pm 0.81$ & 0.008 \\
\hline Social function & Social network (points) & $21.0(7.0)$ & $20.0(11.0)$ & 0.726 & $3.17 \pm 2.58$ & $-4.63 \pm 4.05$ & 0.324 \\
\hline
\end{tabular}

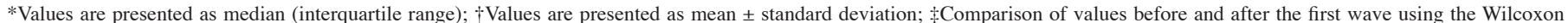
rank-sum test; §Changes in individual physical parameters were assessed using the Student t-test and Mann-Whitney U test; Abbreviations: ASM: Appendicular skeletal muscle, ASMI: Appendicular skeletal muscle index

Table 2. Association between changes in social participation and physical functioning in older women

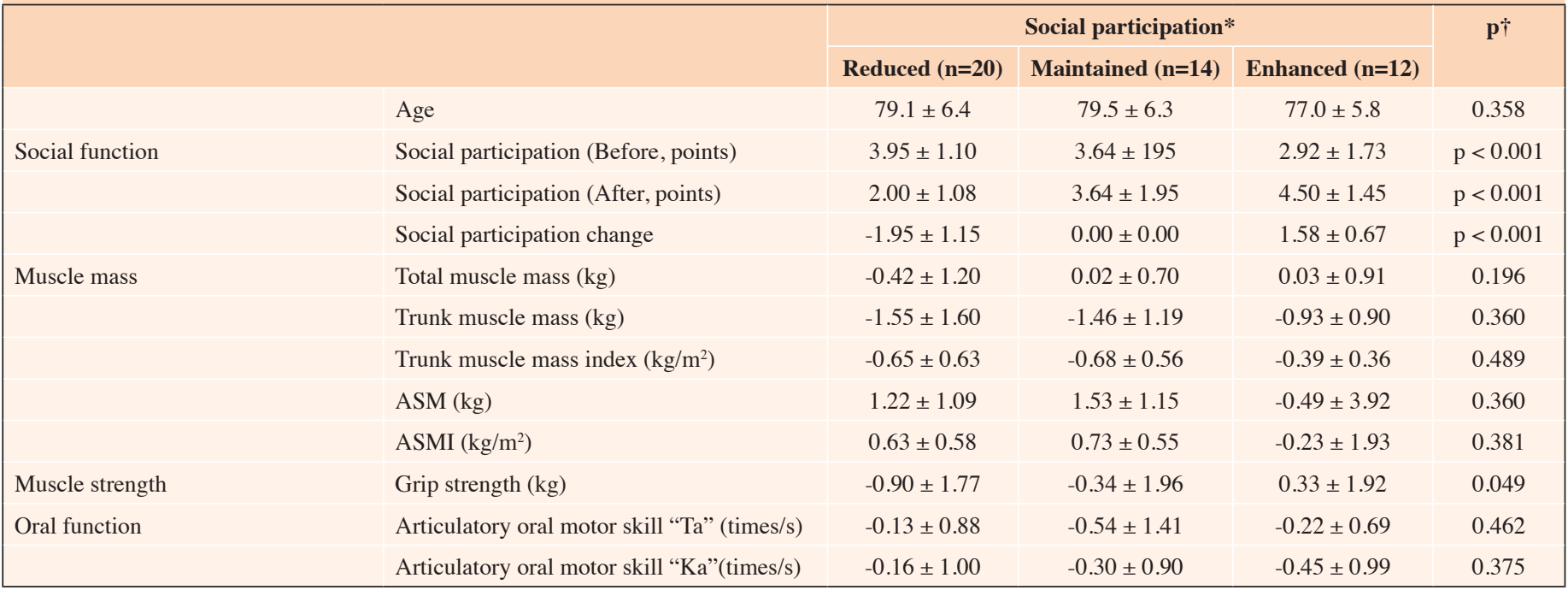

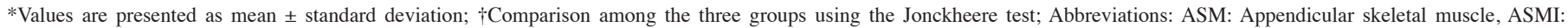
Appendicular skeletal muscle index

Furthermore, changed levels of social participation $(-1.95 \pm$ 1.15 points $)$ and social support $(-1.50 \pm 1.00$ points $)$ in the reduced group were significant compared with the enhance/ maintained group $(0.73 \pm 0.92$ and $0.15 \pm 0.26)$, respectively; thus, implicating that social participation and support were more vulnerable to decrease due to COVID-19 than social network was.

Intriguingly as shown in Table 2, across older women who experienced different levels of social participation (enhanced, maintained, and reduced groups) during the first wave of the COVID-19 pandemic, the changes in grip strength were significant. In addition, total muscle mass and trunk muscle mass values followed similar trends, but significant changes were not seen. Contrarily, among the three groups of social support, there was no difference in muscular measures. Thus, these results demonstrated the importance of social participation even during COVID-19 to prevent sarcopenia and frailty.

\section{Discussion}

In this study, we found that COVID-19-related social detachment resulted in significant declines in trunk muscle mass and social support. Furthermore, when comparing changes in each muscular parameter after the COVID-19 pandemic, we found that trunk muscular mass, grip strength, and oral motor skill "Ka" proficiency were significantly lower in individuals of the reduced group than in enhanced or maintained groups.

Consistent with our results, it has been suggested that sudden activity reduction and increased sedentarism associated with COVID-19 safety measures closely mirror the "catabolic crisis" 
model of sarcopenia. This model suggests that sarcopenia is not simply a gradual process, and is instead accelerated by periodic occasions of inactivity such as periods of extended bed rest or hospitalization. In particular, trunk muscle weakness and imbalance have been implicated as risk factors for postural instability, falls, and poor postoperative outcomes. Furthermore, trunk muscle weakness has been suggested as a cause of walking speed decline in older adults with sarcopenia (15). In further analysis with the men should be examined, since preliminary, a similar tendency was seen before and after the first wave of COVID-19 in trunk muscle mass (9.47 vs. 8.55 $\left.\mathrm{kg} / \mathrm{m}^{2}\right)$, grip strength (30.6 vs. $30.0 \mathrm{~kg}$ ), and oral motor skill ' $\mathrm{Ka}$ ' (6.5 vs. 5.7 times/s) in older four men (mean age, $76.5 \mathrm{y}$; range, 71-80 y).

Recent research assessing the relationship between sarcopenia and trunk muscle strength (16) has revealed that trunk muscle strength weakness is caused by aging, disease, and disuse due to inactivity (17-21). However, unexpectedly, the present study revealed ASM and ASMI significantly increased after the first wave of the COVID-19 pandemic. However, this statistical significance was disappeared when after stratifying the groups according to the changes. Although no precise reasons for ASM and ASMI changes were determined, further investigation is needed with large sample size.

We also found that the levels of oral motor skill decrease due to inactivity, especially "Ka" proficiency, were significant, similar to observations regarding trunk muscle mass and grip strength. Correspondingly, we recently suggested that poor oral health, defined as oral frailty, was significantly associated with increased physical frailty risk, sarcopenia, disability, and mortality in community-dwelling older adults (22). Furthermore, the oral motor skill "Ka" has been suggested to be significantly related to one-leg standing time, handgrip strength (23), bite force, and number of remaining teeth $(24,25)$.

In the present study, we also found that reduction of trunk muscular mass, grip strength, and oral motor skill "Ka" proficiency was not independent, $23.9 \%$ of participants was observed reduction of three all parameters and $43.5 \%$ reduced two of three parameters, suggesting the reciprocal effects of muscle mass, strength, and function.

Social detachment due to COVID-19 is considered a risk factor for health problems in older adults (6). In the present study, we examined the association of social participation during the first wave of COVID-19 with muscular measures. Older women who maintained social participation showed lesser decline in grip strength than those who decreased social participation. Intriguingly, older women with increased levels of social participation had increased grip strength, suggesting that social participation may be an immutably important factor that influences the maintenance or enhancement of muscle strength in community-dwelling older adults. Similarly, a recent study suggested that maintaining social networks increased the odds of maintaining sufficient physical activity in frail older community-dweller during the COVID-19 pandemic (26). Furthermore, in older adults, reduced social interaction due to COVID-19 pandemic was associated with loneliness and depression (4). Moreover, community-dwelling older adults who were less satisfied with important activities were at a higher risk of exhibiting depressive symptom (27), implying the importance of maintaining certain activities, such as hobbies and interpersonal interaction, despite the COVID-19 pandemic. To encourage social participation during ongoing COVID19 restrictions, digital communication, weekly telephone calls, and even a simple exchange of greetings with neighbors are suggested (28). Supporting this, internet-based assistive technologies have been developed and reported to significantly reduce loneliness and increase social support and well-being in older adults (29).

The present study has both strengths and limitations. A strength of the study was the measurement of physical parameters both before (October 2019-February 2020) and after (July-September 2020) the first wave of the COVID-19 pandemic in Japan. This data set may be used to continuously monitor deleterious effects of the COVID-19 pandemic on physical function and may facilitate further investigation of the beneficial effects of social participation. Several limitations of this study should also be addressed. First, the study had a small sample size. Second, there were no morbidity data, nor were numbers of medications recorded.

In conclusion, this study found an impact of the COVID19 on muscular measures, especially significant decreases in trunk muscle mass, grip strength, and oral motor "Ka" proficiency values were observed in older women. Furthermore, older women with enhanced or maintained social participation throughout the first wave of the COVID-19 pandemic had greater grip strength values than those with decreased levels of social participation. This finding indicates that a possible strategy for sarcopenia and frailty prevention is the maintenance of social participation, even during the current pandemic.

Acknowledgements: BK.S. analyzed data and wrote the manuscript. T.I., T.I., and T.N. collected the data. T.T. interpreted the data. K. I supervised the project. All authors discussed results and approved the final manuscript. The authors acknowledge all participants of this study.

Funding: This work was supported by Grants-in-Aid for Scientific Research from the Ministry of Education, Culture, Sports, Science and Technology of Japan (\#204800000032)

Conflicts of Interest: All authors declare that they have no conflicts of interest.

Ethical standards: This study is in accordance with the international ethical standards of research and with the Helsinki Declaration.

\section{References}

1. Tay MZ, Poh CM, Rénia L, et al. The trinity of COVID-19: immunity, inflammation and intervention. Nat Rev Immunol 2020;20:363-374.

2. López J, Perez-Rojo G, Noriega C, et al. Psychological well-being along older adults during the COVID-19 outbreak: a comparative study of the young-old and the old-old adults. Int Psychogeriatr 2020;32:1365-1370.

3. Yamada M, Kimura Y, Ishiyama D, et al. Effect of the COVID-19 epidemic on physical activity in community-dwelling older adults in Japan: A cross-sectional online survey. J Nutr Health Aging 2020;24:948-950.

4. Krendl AC, Perry BL. The Impact of Sheltering in Place During the COVID-19 Pandemic on Older Adults' Social and Mental Well-Being. J Gerontol B Psychol Sci Soc Sci. 2021;76:e53-e58.

5. Yamada M, Kimura Y, Ishiyama Y, et al. The influence of the COVID-19 pandemic on physical activity and new incidence of frailty among initially non-frail older adults in Japan: a follow-up online survey. J Nutr Health Aging 2021;25:751-756.

6. Shimokihara S, Maruta M, Hidaka Y, et al. Relationship of Decrease in Frequency of Socialization to Daily Life, Social Life, and Physical Function in CommunityDwelling Adults Aged 60 and Over after the COVID-19 Pandemic. Int J Environ Res Public Health. 2021;18:2573. 
7. Hironaka S, Kugimiya Y, Watanabe Y, et al. Association between oral, social, and physical frailty in community-dwelling older adults. Arch Gerontol Geriatr 2020;89: 104105.

8. Ishii S, Tanaka T, Shibasaki K, et al., Development of a simple screening test for sarcopenia in older adults. Geriatr Gerontol Int. 2014;14 Suppl 1:93-101.

9. Lee SC, Wu LC, Chiang SL, et al., Validating the Capability for Measuring AgeRelated Changes in Grip-Force Strength Using a Digital Hand-Held Dynamometer in Healthy Young and Elderly Adults. Biomed Res Int. 2020:6936879.

10. Yamada A, Kanazawa M, Komagamine Y, et al. Association between tongue an lip functions and masticatory performance in young dentate adults. J Oral Rehabil 2015;42:833-839.

11. Ito $\mathrm{K}$, Yoshihara A, Takano N, et al. A comparison of methods for the measurement of oral diadochokinesis. Ronen Shika Igaku 2009;24:48-54.

12. Gray J, Kim J, Ciesla JR, Yao P. Rasch analysis of the Lubben Social Network Scale6 (LSNS-6). J Appl Gerontol 2016;35:508-528.

13. Fujita K, Fujiwara Y, Chaves PH, et al. Frequency of going outdoors as a good predictors for incident disability of physical function as well as disability recovery in community-dwelling older adults in rural Japan. J of Epidemiology 2006;16:261-270.

14. Kono A, Kai I, Sakato C, et al. Frequency of going outdoors predicts long-range functional change among ambulatory frail elders living at home. Arch Gerontol Geriatr 2007; 45:233-242.

15. Kato K, Hatanaka Y. The influence of trunk muscle strength on walking velocity in elderly people with sarcopenia. J Phys Ther Sci 2020;32:166-172.

16. Toyoda $\mathrm{H}$, Hoshino M, Ohyama $\mathrm{S}$, et al. The association of back muscle strength and sarcopenia-related parameters in the patients with spinal disorders. Eur Spin J 2019;28:241-249.

17. Kato K, Hatanaka Y, Saito K. The influence of sarcopenia on walking in frail elderly people. OAJ Gerontol Geriatr Med 2017;2:1-6.

18. Mika A, Unnithan VB, Mika P. Difference in thoracic kyphosis and in back muscle strength in women with bone loss due to osteoporosis. Spine 2005;30:241-246.
19. Ito S. Pain and muscle strengthening: about low back pain and trunk muscle strength. Phys Ther J 1998;32:847-854.

20. Tanimoto $\mathrm{Y}$, Watanabe M, Kono R, et al. [Aging changes in muscle mass of Japanese] Nippon Ronen Igakkai Zasshi 2010;47:52-57 (in Japanese).

21. Ikezoe T, Mori N, Nakamura M, et al. Effects of age and inactivity due to prolonged bed rest on atrophy of trunk muscles. Eur J Appl Physiol 2012;112:43-48.

22. Tanaka T, Takahashi K, Hirano H, et al. Oral frailty as a risk factor for physical frailty and mortality in community-dwelling elderly. J Gerontol A Biol Sci Med Sci 2018;73:1661-1667.

23. Izuno H, Hori K, Sawada M, et al. Physical fitness and oral function in communitydwelling older people: a pilot study. Gerondontology 2016;33:470-479.

24. Iinuma T, Arai Y, Fukumoto M, et al. Maximum occlusal force and physical performance in the oldest old: The Tokyo oldest old survey on total health. J Am Geriatr Soc 2012;60:68-76.

25. Yoshida M, Kikutani T, Okada G, et al. The effect of tooth loss on body balance control among community-dwelling elderly persons. Int J Prosthodont 2009;22:136139.

26. Pérez LM, Castellano-Tejedor C, Cesari M, et al. Depressive Symptoms, Fatigue and Social Relationships Influenced Physical Activity in Frail Older CommunityDwellers during the Spanish Lockdown due to the COVID-19 Pandemic. Int J Environ Res Public Health. 2021;18:808.

27. Maruta M, Makizako H, Ikeda Y, et al. Associations between Depressive Symptoms and Satisfaction with Meaningful Activities in Community-Dwelling Japanese Older Adults. J Clin Med. 2020;9:795

28. Banerjee D, Rai M. Social isolation in Covid-19: The impact of loneliness. Int J Soc Psychiatry 2020;66:525-527.

29. Czaja SJ, Boot WR, Charness N, et al. Improving Social Support for Older Adults Through Technology: Findings From the PRISM Randomized Controlled Trial Gerontologist. 2018;58:467-477.

How to cite this article: B.K. Son, T. Imoto, T. Inoue, et al.Social Detachment Influenced Muscle Mass and Strength during the COVID-19 Pandemic in Japanese CommunityDwelling Older Women. J Frailty Aging 2022;11(2)231-235; http://dx.doi.org/10.14283/ jfa.2022.4 